\title{
The BP Network Classification Leafminer-infected Leaves Based on the Fractal Dimension
}

\author{
Dake $\mathrm{Wu}^{1, \mathrm{a}}$, Chunyan $\mathrm{Xie}^{1, \mathrm{~b}}$ \\ ${ }^{1}$ School of Engineering and Technology, Southwest University, Chongqing, P. R. China \\ a keda5@swu.edu.cn (Corresponding author), ${ }^{b}$ kuaileyanyan@126.com
}

Keywords: fractal dimension, BP Network, image processing, leafminer

\begin{abstract}
Leafminer is one of pest of many vegetables, and the damage may cover so much of the leaf that the plant is unable to function, and yields are noticeably decreased. In order to get the information of the pest in the vegetable before the damage was not serious, this research used a BP neural network to classify the leafminer-infected tomato leaves, and the fractal dimension of the leaves was the input data of the BP neural network. Prediction results showed that when the number of FD was 21 and the hidden nodes of BP neural network were 21, the detection performance of the model was good and the correlation coefficient (r) was 0.836. Thus, it is concluded that the FD is an available technique for the detection of disease level of leafminer on tomato leaves.
\end{abstract}

\section{INTRODUCTION}

The fractal dimension (FD) provides a quantitative index of the roughness of natural surfaces, and many other natural phenomena. It has been applied to many different areas in science and engineering such as graphical simulation of clouds or trees ${ }^{[1]}$, texture analysis of some images and material surfaces ${ }^{[2]}$, detection the brain $\mathrm{MRI}^{[3]}$, et al. Automatic ways, such as machine vision ${ }^{[4]}$ and artificial neural networks ${ }^{[5]}$ have been used to detect and recognise the parameters of agricultural pests and diseases.

Leafminer (Liriomyza sativae Blanchard) is a pest of many kinds of vegetable and ornamental plants, such as tomatoes, beans, peas, and various cucurbits. It had been spread out of the country since the first outbreak in China occurred in $1993^{[6]}$. The damage may cover so much of the leaf that the plant is unable to function, and yields are noticeably decreased. In 1995, the leafminers caused a $30 \% \sim 40 \%$ reduction of output in somewhere of China ${ }^{[7]}$. In the past, many researches were found that the spectral range from 737 to $925 \mathrm{~nm}$ was useful for discriminating levels of infestation, especially at wavelengths 755 and $1400 \mathrm{~nm}$, where the differences in reflectance between levels were the largest. Wu et al found that the wavelengths $(771,821,891,945,1026$, $1121,1256,1674,1687$ and $1933 \mathrm{~nm}$ ) related with the damaged degree of the leafminer-infected leaves $^{[8,9]}$, and $\mathrm{Wu}$ et al. used the vis-near infrared spectra to detect gray mold of tomato leaves, they find when the number of primary PCs was 8 and the hidden nodes of BP neural network were 11 , the detection performance of the model was good as correlation coefficient ( $r$ ) was $0.930^{[10]}$.

In the past, chemical sprays have always been used against leafminers due to their protection within the plant. However, the layman often confuses leafminer damage with other leaf diseases and also does not know how bad the damage is. Thus, our objectives were to use leaf image data to determine the leaf damaged degree and the FD in diseased plants, and use the BP network to classify FD data of the tomato leaves.

\section{MATERIALS AND METHODS}

\subsection{The BP network}

The BP network, due to their excellent ability of non-linear mapping, generalization, self-organization and self-learning, has been proved to be of widespread utility in solving complex problems like pattern recognition and adaptation. The training of a BP network usually adopts a back-propagation algorithm, which is a kind of generalized form of the least-mean-squares 
algorithm usually used in engineering. By using the algorithm-generalized gradient descent search technique, the back-propagation algorithm adjusts the weights of the network and the threshold of each neuron recurrently according to the criterion that the cost function is minimized.

\subsection{Calculating the FD of the tomato leaves}

Fractal feature is based on the fractional Brownian motion (FBM) model, which is a technique that combines both fractal and multi resolution image decomposition. The FBM is a part of the set of $1 /$ $\mathrm{f}$ processes, which are the generalization of the ordinary Brownian motion $X(0)$. The FBM is non-stationary, zero-mean Gaussian random functions, which are defined as:

$X(0)=0$

$P(X(t+\Delta r)-X(t) \leq x)=(2 \pi)^{\frac{1}{2}}(\Delta r)^{-H} \int_{-\infty}^{x} \exp \left[\frac{-u^{2}}{2(\Delta r)^{2 H}}\right] d u$

Where $0<H<1$ is the Hurst coefficient that characterizes the FBM. $t$ represent different observation times of the process $X$.

From the Eq. (2), the increment $X(t+\Delta r)-X(t)$ is steady, and its variance between the $\Delta r^{2 H}$ is in proportion, that is:

$E\left[\left((X(t+\Delta r)-X(t))^{2}\right]=k \Delta r^{2 H}\right.$

Where the $H$ is the Hurst coefficient. Falconer ${ }^{[11]}$ had proved that the Hurst coefficient is $2-\mathrm{H}$ when the FBM is curve and $3-\mathrm{H}$ as the FBM is the curved surface. So, in order to compute the FD successfully involves estimation of $H$. Wu et al ${ }^{[7]}$ used a novel computational modeling for estimating $H$. This paper also used this method to estimate the $H$ value, and hence the FD,

\subsection{Materials}

The larvae of the leafminers were collected at the suburb, Beijing, China. And reared on potted tomato seedlings and honeys placed in cages $(50 \mathrm{~cm}$ length $\times 50 \mathrm{~cm}$ width $\times 50 \mathrm{~cm}$ height $)$ fitted with $0.4 \times 0.4 \mathrm{~mm}$ mesh size insect-proof screens, at $\mathrm{T}=27 \pm 1 \mathrm{oC}, \mathrm{RH}=70 \% \pm 5 \%$. Tomatoes were seeded in the 72-cell plug tray, and transplanted in plastic pots of $12 \mathrm{~cm}$ diameter and $10 \mathrm{~cm}$ height with one plant per pot, when the first leaf outspreaded. The pots were placed in a greenhouse (Greenhouse conditions were kept at: $25 / 20^{\circ} \mathrm{C}$ (winter), $30 / 25^{\circ} \mathrm{C}$ (summer); $70 \% \mathrm{RH}$; a photoperiod of $14 \mathrm{~h}$ light: $10 \mathrm{~h}$ dark.) to culture, watered and fertilized regularly to guarantee the plants to growth well. After the third leaf had outspreaded, the plants were placed in the screen cages containing a colony of adult leafminers which had hatched out already one day to infect leaves. 10 seedling pots were placed in the screen cage. After 12 hours, the pots were moved in the greenhouse to culture. 10 infected leaves were collected every time.

\subsection{Computing the damaged degrees and the FD}

The leaf image was taken by the Casio EX-Z3 camera in the natural light, and then processed to grey image, segmented and calculated the total leaf areas and the damaged leaf areas (it is shown in Fig. 1). At last, the damaged degree (DD) of the leave was worked out by using the followed equation:

$\mathrm{DD}=($ The total leaf areas - The damaged leaf areas $) /$ The total leaf areas

The software Matlab 6.5 was used to compute $H$ values with self-written programs, and then the FD. Then the FD values of different leaves were classified by the BP neural network. 

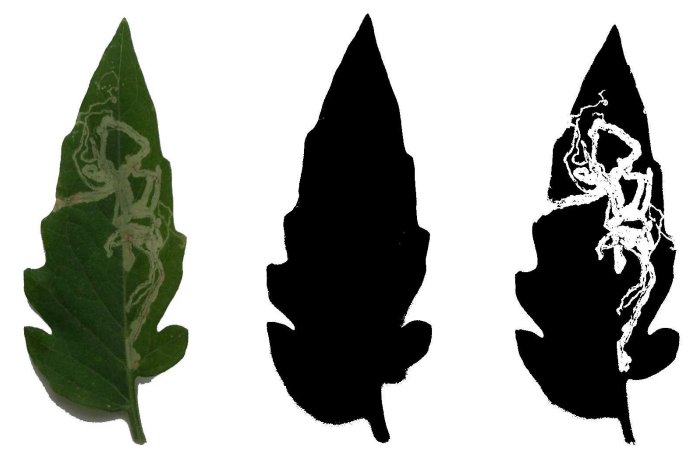

Fig. 1 Tomato leaf image segmentations

(a. Original image; b. Image after segmented (the threshold was 137); c. Image excluded the damaged areas (the threshold was 75))

\section{RESUltS AND DiscuSSIONS}

\subsection{H values and the FD of the leafminer -infected tomato leaves}

When the leaves were infected by the leafminers, the leaf surface was damaged because they ate leaf mesophyll tissues (the leaf lost its chlorophyll, water, and other materials). From the Fig. 2, it is found that the $H$ values are different with different computing steps. At some computing steps, the $H$ values of the leaves are significantly different, so we can use the $H$ values to classify the damaged leaves.

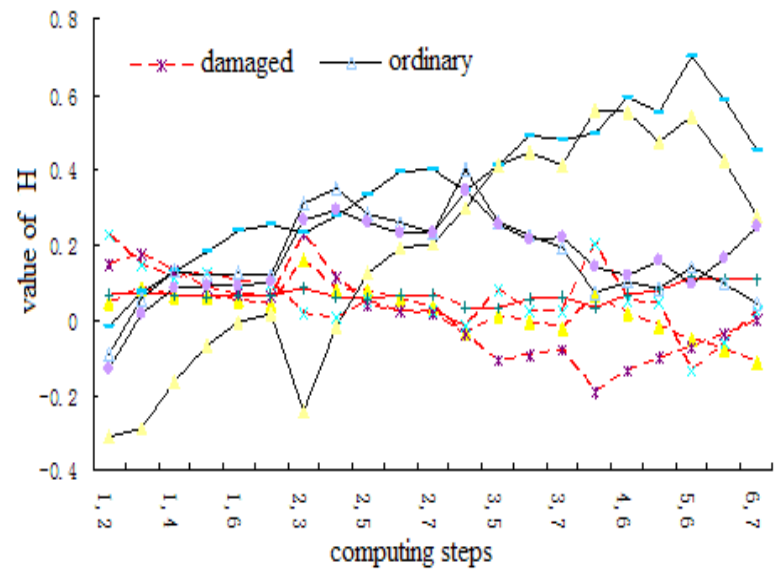

Fig.2 Hurst values of the tomato leaves with different computing steps

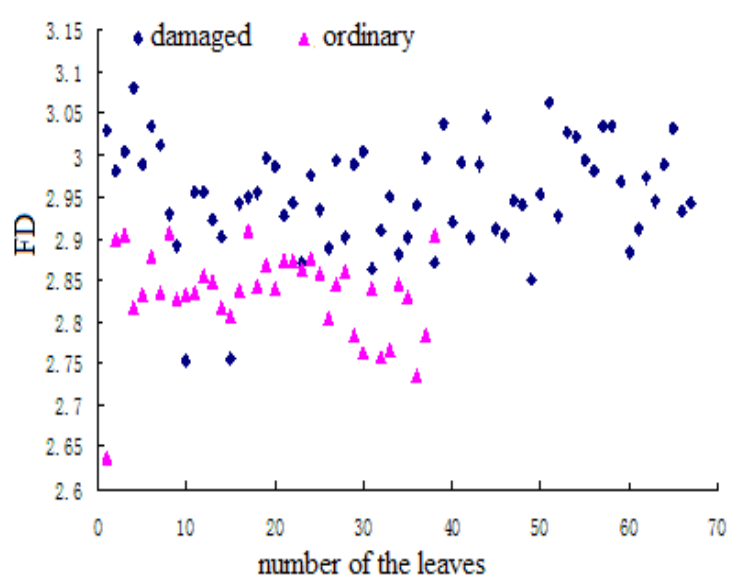

Fig.3 Fractal dimensions of tomato leaves

It is showed that the FD of the leafminer-infected leaves is different from the Fig. 3. The FD between the ordinary and the damaged leaf images has many intersections, so it is hard to classify by using theTM, while the TM can classify the leaves at some computing steps.

\subsection{Thresholds classification (TM) based on the FD}

The TM is good at the linear problem. From the table 2, the classification precision of the TM is the best $(75 \%)$ at the $(3,7)$ computing steps, and is the worst $(35 \%)$ at the $(3,4)$ computing steps. In a word, the classification precision is very low. So, at that step, the leaves can not be classified. Therefore, the nonlinear methods must be used to classify. 
Tab. 2 Classification results of threshold methods based on the tomato leaf fractal dimensions

\begin{tabular}{lcccc}
\hline Steps & $\begin{array}{c}\text { Thresholds } \\
\text { H values }\end{array}$ & $\begin{array}{c}\text { Correct } \\
\text { number }\end{array}$ & $\begin{array}{c}\text { Error } \\
\text { number }\end{array}$ & $\begin{array}{c}\text { Classification } \\
\text { Precision \% }\end{array}$ \\
\hline 2,4 & 0.1494 & 66 & 9 & 50 \\
2,5 & 0.1561 & 66 & 9 & 63 \\
2,6 & 0.1823 & 69 & 6 & 63 \\
2,7 & 0.1832 & 70 & 5 & 50 \\
3,4 & 0.2297 & 70 & 5 & 25 \\
3,5 & 0.2067 & 68 & 7 & 50 \\
3,6 & 0.2349 & 67 & 8 & 63 \\
3,7 & 0.2267 & 69 & 6 & 75 \\
\hline
\end{tabular}

\subsection{The BP network classification based on the FD}

The BP network is a powerful method for pattern recognition and is good at the nonlinear problems. The BP network has been applied as the recognising and classifying system. Fig. 4 was showed the results of the performance of the square errors of the BP network, and the NMSE is convergent. When the number of FD was 21 and the hidden nodes of BP neural network were 21, From the Fig.5, it was found that the recognition provisions were higher (the precisions were $83.6 \%$ ) than the TM's.

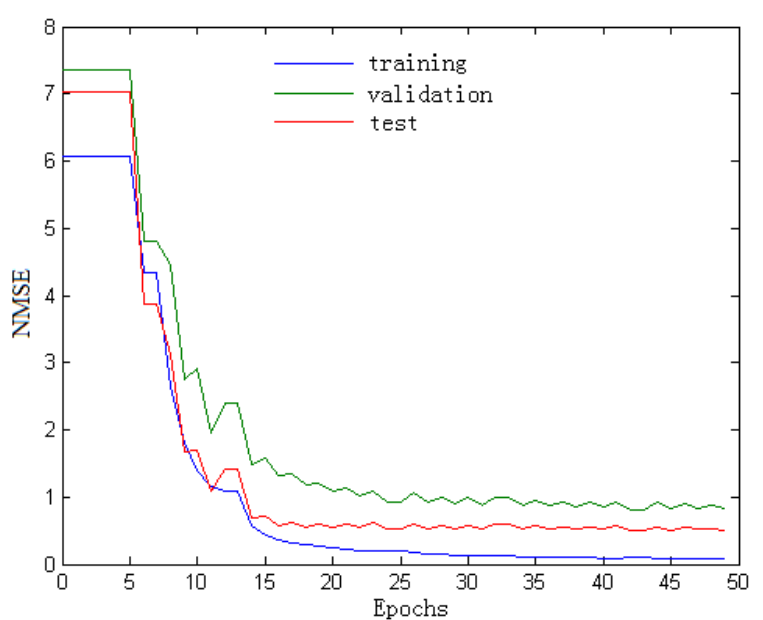

Fig. 4 The square errors of the BP network

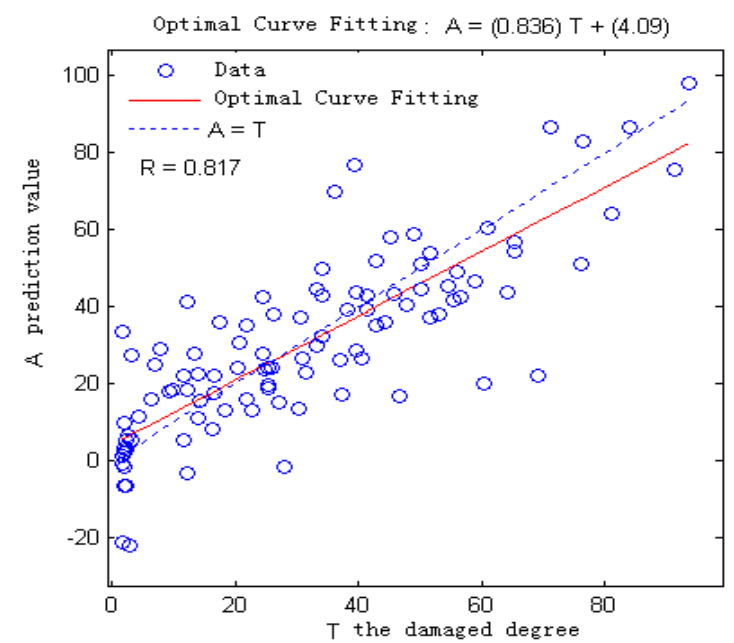

Fig. 5 The output of the BP network

\section{Conclusions}

The paper has presented the FD of the leafminer-infected leaves recognising applications based on the TM and the BP network.

The proposed method has been tested on the samples. Two different kernel functions have been considered to set up the classifying models, and the FD of the damaged tomato leaf image was applied to the TM and the BP network performing of the recognition and classification. The classification precision of the TM is the best $(75 \%)$ at the $(3,7)$ computing steps, and is the worst $(35 \%)$ at the $(3,6)$ computing steps. The precisions of the BP network were 0.836 when the number of the FD was 21 and the hidden nodes of BP neural network were 21. From the experiment, we can use the BP network-based FD models to classify the tomato leaves infected by the leafminers.

\section{ACKNOWLEDGMENTS}

The authors gratefully acknowledge the financial supported by the Fundamental Research Funds for the Central Universities (Grant No. XDJK2009C141 and XDJK2010C048). 


\section{REFERENCES}

[1] B.B. Mandelbrot. The fractal geometry of nature. San Francisco, CA: Freeman, 1982.

[2] J. J. Gagepain, J Groslambert, R Brendel. The fractal dimension of phase and frequency noise: another approach to oscillator characterization. Proceedings of the 39th Annual Frequency Contr. Symposium Philadelphia, PA, May 1985, p. 31-9.

[3] K. Uemuraa, H. Toyamab, and S. Baba1, et al. Generation of fractal dimension images and its application to automatic edge detection in brain MRI. Computerized Medical Imaging and Graphics,2000,24:73-85

[4] D. Y. Qiu, Ch. H. Zhang, H. T. Zhang, et al.. "Application of neural networks in the recognition of stored-grain pests". Transactions of the CSAE, 2003, 19 (1): 142-144 (in Chinese)

[5] J. J. Chen, Sh. W. Ji, J. Li, et al.. "Automatic measurement of danger degree of cotton insect pests using computer vision" Transactions of the CSAE, 2001, 17 (2): 157-160 (in Chinese)

[6] Y.X. Zhao and L. Kang. "Cold tolerance of the leafminer Liriomyza sativae (Dipt., Agromyzidae)”. J. Appl. Ent. 2000, 124: 185-189

[7] D. W. Wei. "Actualities of the Liriomyza sativae Blanchard in China”. Guang xi nong ye ke xue, 2000, 6: 320-324 (in Chinese)

[8] D. K. Wu and C. W. Ma. The support vector machine (SVM) based near-infrared spectrum recognition of leaves infected by leafminers. In: Proceedings of 2006 International Conference on Innovative Computing, Information and Control. VOL 3: 448-451

[9] D. K. Wu, C. W. Ma, S. F. Du. Classification Visible Spectra of Leafminer-Infected Leaves By Support Vector Machine. Transactions of the Chinese Society of Agricultural Machinery,2007, 38(10): 87 90 (in Chinese)

[10] D Wu, L Feng, C Q Zhang, Y He. Study on the Detection of Gray Mold of Tomato Leave Based on Vis-Near Infrared Spectra, Spectroscopy and Spectral Analys , 2007, 27(11): 2208-2211

[11] K. Falconer. Fractal Geometry: Mathematical Foundations and Applications[M]. New York: Willey, 1990 